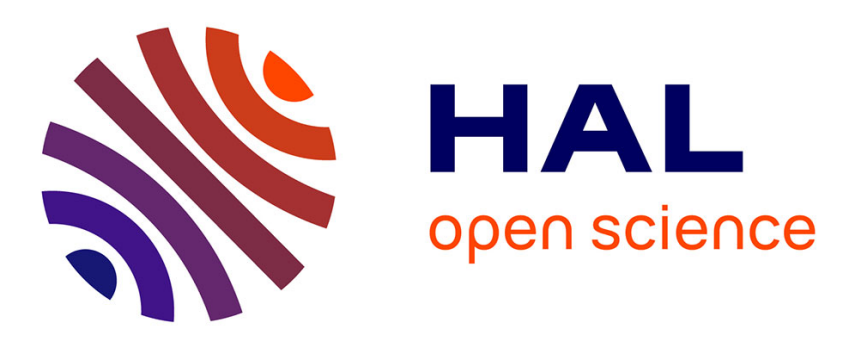

\title{
Modeling rewards and incentive mechanisms for Delay Tolerant Networks
}

Olivier Brun, Rachid El-Azouzi, Balakrishna Prabhu, Tatiana Seregina

\section{To cite this version:}

Olivier Brun, Rachid El-Azouzi, Balakrishna Prabhu, Tatiana Seregina. Modeling rewards and incentive mechanisms for Delay Tolerant Networks. 12th International Symposium on Modeling and Optimization in Mobile, Ad Hoc, and Wireless Networks (WiOpt 2014), May 2014, Hammamet, Tunisia. pp.233 - 240, 10.1109/WIOPT.2014.6850304 . hal-01462314

\author{
HAL Id: hal-01462314 \\ https://hal.laas.fr/hal-01462314
}

Submitted on 8 Feb 2017

HAL is a multi-disciplinary open access archive for the deposit and dissemination of scientific research documents, whether they are published or not. The documents may come from teaching and research institutions in France or abroad, or from public or private research centers.
L'archive ouverte pluridisciplinaire HAL, est destinée au dépôt et à la diffusion de documents scientifiques de niveau recherche, publiés ou non, émanant des établissements d'enseignement et de recherche français ou étrangers, des laboratoires publics ou privés. 


\title{
Modeling Rewards and Incentive Mechanisms for Delay Tolerant Networks
}

\author{
O. Brun ${ }^{\star}$, R. El-Azouzi ${ }^{\dagger}$, B. Prabhu ${ }^{\star}$, T. Seregina ${ }^{\star}$
}

\begin{abstract}
A central problem in Delay Tolerant Networks (DTNs) is to persuade mobile nodes to participate in relaying messages. Indeed, the delivery of a message incurs a certain number of costs for a relay. We consider a two-hop DTN in which a source node, wanting to get its message across to the destination as fast as possible, promises each relay it meets a reward. This reward is the minimum amount that offsets the expected delivery cost, as estimated by the relay from the information given by the source (number of existing copies of the message, age of these copies). A reward is given only to the relay that is the first one to deliver the message to the destination. For two relays and exponentially distributed inter-contact times, we show that the expected reward the source pays remains the same irrespective of the information it conveys, provided that the type of information does not vary dynamically over time. On the other hand, the source can gain by adapting the information that it conveys to a meeting relay.
\end{abstract}

\section{INTRODUCTION}

The core objective for the design of Delay Tolerant Networks (DTNs) is to support communications even when endto-end connectivity fails. This might happen either due to mobility of nodes or because communication devices become unreachable for a large period of time. In those cases, DTNs can exchange data in spite of the lack of a dedicated network infrastructure. In practice, DTNs are composed of mobile devices, including smartphones, tablets or other mobile devices having multiple wireless interfaces. For such devices, OS APIs are available to program dedicated applications for direct data exchange with peer nodes during radio range contacts. The networking community has mainly focused on how to route messages reliably towards the intended destination(s). Replication of the original message by the so-called epidemic routing protocol ensures that at least some copy will reach the destination node with high probability and with a minimum delivery delay. Another standard optimization problem is how to maximize the delivery probability under constraints on the resources spent to forward the message to the destination [1], [2]. Several papers have further extended the possible optimization to the use of activation and/or forwarding control at relays [3], [4], [5]. Almost all earlier works on modelling performance of DTNs have assumed that relays in DTN are willing to cooperate with source nodes. However, a central question is whether owners of relay devices are willing to have battery depleted to sustain DTNs communications. The selfish behavior of relays becomes a core threat which hinders any possible attempt to optimize network performance.

^LAAS/CNRS; Université de Toulosue, 7, avenue du Colonel Roche, 31031 Toulouse, France. ${ }^{\dagger}$ CERI/LIA, University of Avignon, 339, chemin des Meinajaries, Avignon, France.
In different contexts, user participation in network operations is assured by means of appropriate incentive mechanisms. In peer-to-peer systems, many works proposed a reward mechanism for providing incentives for cooperation in order to discourage free riders. In the case of DTNs, nevertheless, there is an additional technical issue to be solved. In fact, the credit exchange mechanism cannot be based on endto-end communications between nodes due to the fact that feedback messages in DTNs may incur large delays. In order to avoid using feedback messages, we introduce a rewarding mechanism promoting the participation of nodes as relays. This scheme mandates that only a successful relay (i.e., the first relay to deliver a message to the destination) receives a reward. In fact, in order to avoid the use of feedbacks that allow relays to know whether the message has been successfully delivered or not, we assume that a relay will receive a reward if and only if it is the first one to deliver the message to the destination.

Thus, the reward offered is an incentive reward by which the source may persuade a mobile node that it meets to become a relay. To do so, the source has to decide the amount of reward it proposes to each potential relay that it meets, and the relays have to decide whether to accept the message or not. The success of a given relay depends on the number of relays that have already accepted the message: the bigger the number of nodes relaying the message, the higher the delivery probability for the message, but indeed the less the chance for the given relay to receive a reward from the system. Furthermore, we will consider different information settings: full information in which the source informs the meeting relay about the number of relays that have already accepted the message and at which time; partial information in which the source gives to each relay it meets only the information on the number of existing message copies, and no information where the relays do not have any information.

\section{A. Contributions}

In a DTN with two relays, we investigate how the reward that guarantees full cooperation of relays depends on the information that source makes available to relays, and the time instant at which the relays meets the source. If the source does not adapt the information it gives, we show that the average reward paid by source for delivering a message remains the same irrespective of the information it conveys. We then show that the source can do better by changing its strategy on the fly. When it meets the first relay, it is always optimal for the source to tell it that it is first one. With this information the source has 
to pay less to this relay. When it meets the second relay, we show that the information to be given depends on the how late this relay meets the source with respect to the first relay. After a certain threshold, it is optimal for the source not the give any information whereas before this threshold it is optimal for the source to give full information, that is tell the relay that is second and also tell the time at which the first relay met the source. The results obtained are for exponentially distributed inter-contact times between the relays and the source as well as between the relays and the destination. This assumption is justified at certain time-scales [6]. While other distributions such as power-law have also been experimentally observed, in this paper we restrict the analysis to that of the exponential distribution.

\section{B. Related works}

Several incentive schemes have been developed for DTNs. For example, [7] uses Tit-for-Tat (TFT) to design an incentiveaware routing protocol that allows selfish DTN nodes to optimize their individual utilities under TFT constraints. Mobicent [8] is a credit-based incentive system which integrates credit and cryptographic technique to solve the edge insertion and edge hiding attacks among nodes. MobiGames [9] is a usercentric and social-aware reputation based incentive scheme for DTNs. In addition, [10] develops socially selfish routing in DTNs, where the decision of a node to participate on forwarding process is based uses social willingness. RELICS [11] is another cooperative based energy-aware incentive mechanism for selfish DTNs, in which a rank metric was defined to measure the transit behavior of a node. In [12], authors proposed an incentive driven dissemination scheme that encourages nodes to cooperate and chooses delivery paths that can reach as many nodes as possible with fewest transmissions. Recently, authors in [13] proposed a rewarding scheme where relays gain certain fixed reward that incentivise them to sacrifice memory and battery on DTNs relaying operation. This mechanism is designed using the theory of Minority Games in order obtain cooperation of relays in distributed fashion. They also proposed a learning algorithm which moves the system to the aforementioned equilibria with just local estimation of system parameter at relay nodes.

The rest of the paper is organized as follows. In Section II, we describe the mobility model, the cost structure for the relays, and the information that the source can give to the relays. In Section III, we compute the expected reward for the source in the three possible information settings. In Section IV, we compute the optimal adaptive strategy for the source and compare it with the static strategy. The conclusions and the future work are summarized in Section V.

\section{SySTEM MODEL}

Consider a network of wireless nodes with one source node, one destination node, and two relays. We shall assume that the source and the destination are fixed whereas the relays are moving according to a given mobility model. The inter- contact time between a relay and the source (resp. destination) is assumed to be $i . i . d$ with density function $f_{s}$ (resp. $f_{d}$ ).

The source generates a message at time 0 and wants it to be delivered to the destination. It cannot do so directly but has to send the message through one of the relays. It is assumed that the network is two-hop, that is a relay cannot forward the message to another relay. It has to deliver the message by itself to the destination. The delivery of a message incurs a certain number of costs for a relay :

1) reception cost, $C_{r}$ : this is a fixed energy cost for receiving the message from the source;

2) storage cost, $C_{s}$ : this is the incurred cost per unit time the message is stored in the relay; and

3) transmission cost $C_{d}$ : this is the fixed cost for transmitting the message to the destination.

In order to offset these costs, the source proposes a reward to the relays, which can decide to either accept or reject the proposition. The source is not informed when the message reaches the destination. Thus, it can propose a reward to the second relay even if the first relay has already delivered the message. In case both the relays accept, then the reward is given only to the relay that is the first one to deliver the message to the destination.

The source has to decide the amount of reward it proposes to each relay, and the relays have to decide whether to accept the message or not. An important assumption we shall make is that a relay will accept the message if the expected reward offsets its expected costs which depend notably on the information given by the source to the relays. Note that the reward the relay gets is a random variable which depends on whether it is the first one to deliver the message - the relay gets nothing if it is not the first one to deliver, otherwise it gets the proposed reward. The expected reward that a relay gets will depend upon the information the source gives to the relay and when the two meet. The source could inform the relay on whether it is the first or the second one, and if the relay is second then the source could choose to tell the instant when the first relay met the source. The dependence of the reward on the information has the following intuitive explanation. The relay that is the second one to meet the source has a smaller probability of getting the reward since the first relay could have already delivered the message to the destination. Since the second relay has a higher risk of failure, it will naturally ask for a higher reward to offset its costs. Here we have assumed that the source tells a relay whether it is first or second. It can choose not to give this information in which case the relay which arrives later will again ask for a higher reward.

From the point of view of the source, the expected reward it pays will depend on the information it gives. We shall investigate how the expected reward to be paid by the source depends on the information. For this we shall consider three settings:

1) no information: the relays are not told whether they are first or second to meet the source. Each relay only knows at what time it meets the source. 
2) partial information: each relay is told whether it is the first or second to meet the source. The second relay is not told when the first one met the source.

3) full information: each relay is told whether it is the first or second. Moreover, the second relay knows at what time the first one met the source.

Which of these settings is better for the source will certainly depend on the mobility model for the relays and the intercontact time distribution. As a first attempt, in this paper we shall restrict the analyses to exponentially distributed intercontact times with parameter $\lambda$ (resp. $\mu$ ) for the source (resp. destination).

\section{EXPECTED REWARD PAID BY THE SOURCE UNDER THE THREE SETTINGS}

Since the source wishes to transmit the message as fast as possible to the destination, it is clear that it has to give the message to any relay it meets, whatever the value of the reward requested by this relay. Our aim is to compare the expected reward the source will pay out in the three settings to determine which is best option for the source. In the sequel, we shall use the superscript $F$ (resp. $P, N$ ) to denote quantities related to the full information (resp. partial information, no information) setting.

\section{A. Expression of the expected reward paid by the source}

Let $k \in\{F, P, N\}$ be one of the three settings. Let $S_{1}$ be the random time at which the message is given by the source to the first relay it meets, and $S_{2}>S_{1}$ be the random time at which the second relay meets the source. For fixed $s_{1}$ and $s_{2}$, we denote by $\mathbf{s}$ the vector $\left(s_{1}, s_{2}\right)$. We define $V_{i}^{(k)}(\mathbf{s})$ as the net cost for relay $i=1,2$ under setting $k$, and we let $R_{i}^{(k)}$ (s) be the reward asked by this relay to the source under this setting. Of course, $V_{1}^{(k)}(\mathbf{s})$ and $R_{1}^{(k)}(\mathbf{s})$ do not depend on $s_{2}$ since it is not known at what time the second relay meets the source. Similarly, $V_{2}^{(k)}(\mathbf{s})$ and $R_{2}^{(k)}(\mathbf{s})$ depend on $s_{1}$ only in the full information setting, that is for $k=F$. As mentioned in Section II, the reward proposed to a relay has to offset its expected cost. The latter depends on the information given by the source to relay $i$ only through the probability of success $p_{i}^{(k)}(\mathbf{s})$ estimated by the relay when it receives the message from the source. We establish below the general expression of the expected reward paid by the source in terms of these estimated probabilities of success.

1) Expected reward of a relay for given contact times: The net expected cost for relay $i$ under setting $k$ is given by

$$
\mathbb{E}\left[V_{i}^{(k)}(\mathbf{s})\right]=C_{r}+C_{s} \frac{1}{\mu}+\left[C_{d}-R_{i}^{(k)}(\mathbf{s})\right] p_{i}^{(k)}(\mathbf{s}) .
$$

The first term in the net expected cost is the reception cost. The second term corresponds to the storage cost. The interval of time from the instant the relay meets the source to the instant when it meets the destination is also exponentially distributed with rate $\mu$. This follows from the memoryless property of the exponential distribution. Hence, the expected duration for which the message is stored is $1 / \mu$, which then engenders an expected storage cost of $C_{s} / \mu$. The final term is the cost of transmitting the message to the destination which then gives the reward to the relay. This term enters into play only if the relay is the first one to contact the destination with the message, which explains the factor $p_{i}^{(k)}(\mathbf{s})$.

Relay $i=1,2$ will accept the message if the proposed reward offsets the cost, that is, if $\mathbb{E}\left[V_{i}^{(k)}(\mathbf{s})\right] \leq 0$. Thus, the minimum reward that the source has to promise relay $i$ is given by

$$
\begin{aligned}
R_{i}^{(k)}(\mathbf{s}) & =C_{d}+\left(C_{r}+C_{s} \frac{1}{\mu}\right) \frac{1}{p_{i}^{(k)}(\mathbf{s})} \\
& =: \quad C_{1}+C_{2} \frac{1}{p_{i}^{(k)}(\mathbf{s})} .
\end{aligned}
$$

2) Expected reward paid by the source for given contact times: Given that $S_{1}=s_{1}$ and $S_{2}=s_{2}$, the expected reward paid by the source is

$$
R_{S}^{(k)}(\mathbf{s})=R_{1}^{(k)}(\mathbf{s})(1-p(\mathbf{s}))+R_{2}^{(k)}(\mathbf{s}) p(\mathbf{s}),
$$

where $p(\mathbf{s})$ is the (real) probability of success of the second relay given $s_{1}$ and $s_{2}$. We now proceed to compute $p(\mathbf{s})$.

Lemma 1: The probability of success of the second relay is given by

$$
p(\mathbf{s})=\frac{e^{-\mu\left(s_{2}-s_{1}\right)}}{2} .
$$

Proof: Let $Y_{1}$ (resp. $Y_{2}$ ) be the first time instant after $s_{1}$ (resp. $s_{2}$ ) when the first (resp. second) relay meets the destination. From the memoryless property of the exponential distribution $\mathbb{P}\left(Y_{1}>t\right)=e^{-\mu\left(t-s_{1}\right)}$. Similarly, $\mathbb{P}\left(Y_{2}>\right.$ $t)=e^{-\mu\left(t-s_{2}\right)}$. Then, given that the second relay meets the destination for the first time after $s_{2}$ at time $t$, its probability of success is

$$
p\left(\mathbf{s} \mid Y_{2}=t\right)=\mathbb{P}\left(Y_{1}>t\right)=e^{-\mu\left(t-s_{1}\right)} .
$$

Unconditioning on $Y_{2}$, we obtain

$$
\begin{aligned}
p(\mathbf{s}) & =\int_{t=s_{2}}^{\infty} p\left(\mathbf{s} \mid Y_{2}=t\right) \mu e^{-\mu\left(t-s_{2}\right)} d t \\
& =\int_{s_{2}}^{\infty} e^{-\mu\left(t-s_{1}\right)} \mu e^{-\mu\left(t-s_{2}\right)} d t \\
& =\frac{e^{-\mu\left(s_{2}-s_{1}\right)}}{2} .
\end{aligned}
$$

3) Expected reward paid by the source: Let $\bar{R}^{(k)}$ denote the expected reward paid by the source under setting $k$. Unconditioning on $S_{1}$ and $S_{2}$, we obtain

$$
\bar{R}^{(k)}=\int_{s_{1}=0}^{\infty} \int_{s_{2}=s_{1}}^{\infty} R_{S}^{(k)}(\mathbf{s}) f_{S_{1}, S_{2}}(\mathbf{s}) d s_{2} d s_{1},
$$

where $f_{S_{1}, S_{2}}\left(s_{1}, s_{2}\right)$ is the joint distribution of $S_{1}$ and $S_{2}$. Since the inter-contact times are exponentially distributed with parameter $\lambda$, the joint distribution of $S_{1}$ and $S_{2}$ is the 
order statistics of two exponentially distributed variables of parameter $\lambda$ [14]. That is,

$$
f_{S_{1}, S_{2}}(\mathbf{s})=2 \lambda^{2} e^{-\lambda\left(s_{1}+s_{2}\right)}, 0 \leq s_{1} \leq s_{2}<\infty .
$$

The resulting expected reward is stated in the following proposition.

Proposition 1: The expected reward paid by the source is expressed in terms of the probabilities of success estimated by the relays as follows

$$
\begin{aligned}
\bar{R}^{(k)} & =C_{1} \\
& +C_{2} \int_{0}^{\infty} \int_{s_{1}}^{\infty}\left(\frac{1-p(\mathbf{s})}{p_{1}^{(k)}(\mathbf{s})}+\frac{p(\mathbf{s})}{p_{2}^{(k)}(\mathbf{s})}\right) 2 \lambda^{2} e^{-\lambda\left(s_{1}+s_{2}\right)} d s_{2} d s_{1}
\end{aligned}
$$

Proof: The proof directly follows from (5), (6), (3) and (2).

We now proceed to the analysis of the probability of success estimated by the relays in the three settings.

\section{B. Full information}

In this setting, once a relay meets the source, it is informed whether it is the first or the second to meet the source, and if the relay is second then it is informed of the time the first one met the source.

1) Minimal reward for the second relay: The second relay knows $s_{1}$ and is therefore in position to compute exactly its probability of success, so that $p_{2}^{(F)}(\mathbf{s})=p(\mathbf{s})$. According to (2), the reward proposed to the second relay is then

$$
R_{2}^{(F)}(\mathbf{s})=C_{1}+C_{2} \frac{1}{p(\mathbf{s})},
$$

where the expression of $p(\mathbf{s})$ is given in (4). Observe that the reward proposed to the second relay depends on $s_{1}$ and $s_{2}$ only through their difference, and increases exponentially with this difference.

2) Minimal reward for the first relay: In a similar way, one can compute the reward proposed to the first relay by first computing its estimated probability of success $p_{1}^{(F)}(\mathbf{s})$.

Lemma 2:

$$
p_{1}^{(F)}(\mathbf{s})=q=1-\frac{\lambda}{2(\lambda+\mu)} .
$$

Proof: The first relay does not know at what time the second relay will meet the source but knows that this time is exponentially distributed with parameter $\lambda$. Let $S_{2}$ denote this random time. From the memoryless property of the exponential distribution, $\mathbb{P}\left(S_{2}>x\right)=e^{-\lambda\left(x-s_{1}\right)}$. Given the second relay meets the source at $s_{2}$, the probability of success of the first relay is $1-p(\mathbf{s})$, which was computed in (4). Now,
$p_{1}^{(F)}(\mathbf{s})$ can be found by unconditioning on $S_{2}$, that is,

$$
\begin{aligned}
p_{1}^{(F)}(\mathbf{s}) & =\int_{x=s_{1}}^{\infty} p_{1}^{(F)}\left(\mathbf{s} \mid S_{2}=x\right) \lambda e^{-\lambda\left(x-s_{1}\right)} d x \\
& =\int_{x=s_{1}}^{\infty}(1-p(\mathbf{s})) \lambda e^{-\lambda\left(x-s_{1}\right)} d x \\
& =\int_{x=s_{1}}^{\infty}\left(1-\frac{e^{-\mu\left(x-s_{1}\right)}}{2}\right) \lambda e^{-\lambda\left(x-s_{1}\right)} d x \\
& =1-\frac{\lambda}{2(\lambda+\mu)} .
\end{aligned}
$$

The reward proposed to the first relay is then

$$
R_{1}^{(F)}\left(s_{1}\right)=C_{1}+C_{2} \frac{1}{q} .
$$

Note that in this setting $p_{1}^{(F)}(\mathbf{s})$ and hence $R_{1}^{(F)}$, in addition to be independant of $s_{2}$ as expected, does not depend on $s_{1}$ but only on the fact that this relay is the first one to meet the source.

3) Expected reward paid by the source: The resulting expected reward is stated in the following proposition.

Proposition 2: Let $\bar{R}^{(F)}$ denote the expected reward paid by the source when it gives full information to the relays. Then,

$$
\bar{R}^{(F)}=C_{1}+2 C_{2} .
$$

Proof: Using the expressions of $R_{1}^{(F)}$ and $R_{2}^{(F)}$ given in (11) and (8), and substituting them in (7)

$$
\begin{aligned}
\bar{R}^{(F)} & =C_{1} \\
& +C_{2} \int_{0}^{\infty} \int_{s_{1}}^{\infty}\left(\frac{1-p(\mathbf{s})}{q}+1\right) 2 \lambda^{2} e^{-\lambda\left(s_{1}+s_{2}\right)} d s_{2} d s_{1} \\
& =C_{1}+C_{2} \\
& +C_{2} \int_{0}^{\infty} \frac{2 \lambda e^{-2 \lambda s_{1}}}{q\left(s_{1}\right)} \int_{s_{1}}^{\infty}(1-p(\mathbf{s})) \lambda e^{-\lambda\left(s_{2}-s_{1}\right)} d s_{2} d s_{1} \\
& =C_{1}+2 C_{2},
\end{aligned}
$$

where the last equality follows from (9).

\section{Partial Information}

In this setting, the source informs a relay whether it is first or second. If the relay is second, the source does not inform the relay of the time at which it met the first relay.

1) Minimal reward for the second relay: Now the second relay does not know when exactly the first relay met the source. Let $S_{1}$ denote this random time. The second relay knows that the distribution of inter-contact times is exponential with parameter $\lambda$. Hence, the distribution of $S_{1}$ given that the second relay meets the source at $s_{2}$ is given by

$$
\mathbb{P}\left(S_{1}<y\right)=\frac{1-e^{-\lambda y}}{1-e^{-\lambda s_{2}}} .
$$

The probability of success estimated by the second relay given that it meets the source at $s_{2}$ is $p_{2}^{(P)}(\mathbf{s})$. Note that 
$p_{2}^{(P)}(\mathbf{s})$ does not depend on $s_{1}$ which is unknown to the second relay. This probability can be computed using the same method as the probabilities in the setting with full information. Hence, we state the result without proof.

$$
\begin{aligned}
p_{2}^{(P)}(\mathbf{s}) & =\int_{y=0}^{s_{2}} p\left(y, s_{2}\right) \frac{\lambda e^{-\lambda y}}{1-e^{-\lambda s_{2}}} d y \\
& =\frac{1}{2} \frac{\lambda}{\lambda-\mu} \frac{e^{-\mu s_{2}}-e^{-\lambda s_{2}}}{1-e^{-\lambda s_{2}}} .
\end{aligned}
$$

Hence, the reward proposed to it by the source has to be

$$
R^{(P)}\left(s_{2}\right)=C_{1}+C_{2} \frac{1}{p_{2}^{(P)}(\mathbf{s})} .
$$

2) Minimal reward for the first relay: In this setting, the first relay obtains the same information from the source as in the setting with full information. As a consequence, the minimal reward that the source should propose to it is the same as in the first setting, and is thus given by

$$
R_{1}^{(P)}\left(s_{1}\right)=R_{1}^{(F)}=C_{1}+\frac{C_{2}}{q} .
$$

3) Expected reward paid by the source: Let $\bar{R}^{(P)}$ be the expected reward to be paid by the source when it gives partial information. It turns out that

Proposition 3:

$$
\bar{R}^{(P)}=C_{1}+2 C_{2}
$$

Proof: Using the expressions of $R_{1}^{(P)}$ and $R_{2}^{(P)}$ given in (16) and (15), and substituting them in (7)

$$
\begin{aligned}
\bar{R}^{(P)} & =C_{1} \\
& +C_{2} \int_{s_{1}=0}^{\infty} \int_{s_{2}=s_{1}}^{\infty} \frac{1-p(\mathbf{s})}{q} 2 \lambda^{2} e^{-\lambda\left(s_{1}+s_{2}\right)} d s_{2} d s_{1} \\
& +C_{2} \int_{s_{2}=0}^{\infty}\left(\int_{s_{1}=0}^{s_{2}} \lambda e^{-\lambda s_{1}} p(\mathbf{s}) d s_{1}\right) \frac{1}{p_{2}^{(P)}(\mathbf{s})} 2 \lambda e^{-\lambda s_{2}} d s_{2} .
\end{aligned}
$$

From (9), the second term is equal to $C_{2}$. That the last term is also equal to $C_{2}$ follows directly from (13).

\section{No information}

In the final setting, a relay does not know whether it is first or second. Since relays have no information on the order of contacts with the source and the time at which the other relay met the source, the reward a relay will ask will depend only on the instant it meets the source.

1) Minimal reward for a relay:

Lemma 3: In the setting with no information, the probability of success of relay $i$ meeting the source at time $s_{i}$ is given by

$$
p_{i}^{(N)}(\mathbf{s})=\frac{\lambda}{\lambda-\mu} \frac{e^{-\mu s_{i}}}{2}-\frac{\mu^{2}}{\lambda^{2}-\mu^{2}} e^{-\lambda s_{i}},
$$

Proof: Let $Y$ be the instant when relay $i$ meets the destination. Let $X$ (resp. $Z$ ) be the instant when the other relay meets the source (resp. destination). Then, relay $i$ gets the reward if either the other relay meets the source after $Y$ or if the other relay meets the source before $Y$ but meets the destination after $Y$, that is,

$$
p_{i}^{(N)}(\mathbf{s})=\mathbb{1}_{\{X>Y\}}+\mathbb{1}_{\{X<Y, Z>Y\}}
$$

From the law of total probability,

$$
\begin{aligned}
p_{i}^{(N)}(\mathbf{s})= & \int_{t=s}^{\infty} \mu e^{-\mu(t-s)} \int_{x=0}^{\infty} \lambda e^{-\lambda x} \int_{z=x}^{\infty} \mu e^{-\mu(z-x)} \\
& p_{i}^{(N)}(\mathbf{s} \mid Y=t, X=x, Z=z) d z d x d t \\
= & \int_{t=s}^{\infty} \mu e^{-\mu(t-s)} \int_{x=0}^{\infty} \lambda e^{-\lambda x} \int_{z=x}^{\infty} \mu e^{-\mu(z-x)} \\
& \left(\mathbb{1}_{\{x>t\}}+\mathbb{1}_{\{x<t, z>t\}}\right) d z d x d t \\
= & \int_{t=s}^{\infty} \mu e^{-\mu(t-s)} e^{-\lambda t} d t \\
+ & \int_{t=s}^{\infty} \mu e^{-\mu(t-s)} \int_{x=0}^{t} \lambda e^{-\lambda x} \int_{z=t}^{\infty} \mu e^{-\mu(z-x)} d z d x d t \\
= & \frac{\lambda}{\lambda-\mu} \frac{e^{-\mu s}}{2}-\frac{\mu^{2}}{\lambda^{2}-\mu^{2}} e^{-\lambda s}
\end{aligned}
$$

which, if $\lambda=\mu$, reduces to $\frac{3+2 \lambda s}{4} e^{-\lambda s}$.

According to (2), the minimal reward that the source should propose to relay $i$ is

$$
R_{i}^{(N)}(\mathbf{s})=C_{1}+C_{2} \frac{1}{p_{i}^{(N)}(\mathbf{s})} .
$$

2) Expected reward paid by the source: Let $\bar{R}^{(N)}$ be the expected reward paid by the source when it gives no information. In this setting as well, the expected reward is the same as in the previous two settings and is

$$
\bar{R}^{(N)}=C_{1}+2 C_{2} \text {. }
$$

The proof follows the same lines as in the full information and partial information settings.

\section{Adaptive Strategy}

It was seen in the previous section that the expected reward paid by the source is the same in the three settings. Thus, if the source uses a fixed strategy independently of $s_{1}$ and $s_{2}$, then it is not beneficial for the source to give the state information to the relays.

We now show that the source can reduce the expected reward to be paid by adapting its strategy on the fly as and when it meets the relays. Since the source knows at what time it met the first relay, it can decide to either withhold or give information to the second relay depending on $s_{1}$ and $s_{2}$. The decision of the source will depend upon the reward it has to propose in each of the three settings.

A key assumption we shall make is that the relays are naive, that is, they do not react to the fact that the source is adapting its strategy. A relay computes its success probability purely based on the information it receives and according to the formulas obtained in the previous section. In practice, if the relay knows that the source will adapt its strategy as a function 
of time, then the relay also must react accordingly. However, as a first model, we assume that the relays are naive.

Our first result in this direction states that it is always beneficial for the source to give information to the first relay independently of $s_{1}$.

Proposition 4:

$$
R_{1}^{(F)}(\mathbf{s})=R_{1}^{(P)}(\mathbf{s}) \leq R_{1}^{(N)}(\mathbf{s})
$$

Proof: The equality $R_{1}^{(F)}=R_{1}^{(P)}$ follows from (11) and (16). So, we now show that $R_{1}^{(F)} \leq R_{1}^{(N)}$. Note that for this it is sufficient to show that

$$
p_{1}^{(N)}(\mathbf{s}) \leq p_{1}^{(F)}(\mathbf{s})=q, \forall s_{1} \geq 0 .
$$

From (18), first note that $p_{1}^{(N)}\left(0, s_{2}\right)=q$. It is thus sufficient to show that the derivate of $p_{1}^{(N)}(\mathbf{s})$ with respect to $s_{1}$ is negative to complete the proof. From (18),

$$
\frac{d}{d s_{1}} p_{1}^{(N)}\left(s_{1}, s_{2}\right)=\frac{\lambda \mu^{2} e^{\mu s_{1}}}{\lambda-\mu}\left(-\frac{e^{-2 \mu s_{1}}}{2 \mu}+\frac{e^{-(\lambda+\mu) s_{1}}}{\lambda+\mu}\right) .
$$

For $\lambda>\mu$, since $e^{-x} / x$ is a decreasing function of $x$, it follows that $-\frac{e^{-2 \mu s_{1}}}{2 \mu}+\frac{e^{-(\lambda+\mu) s_{1}}}{\lambda+\mu}$ seen as a function of $\lambda$ is negative. Hence $p_{1}^{(N)}\left(s_{1}, s_{2}\right)$ is a decreasing function of $s_{1}$. A similar argument shows the same result for $\lambda \leq \mu$.

We now turn our attention to what the source should do when it meets the second relay. First, let us restrict the set of choices to either partial information (that is, the relay is told that it is the second relay but not told the $s_{1}$ ) or no information. Then,

Proposition 5:

$$
R_{2}^{(N)}(\mathbf{s}) \leq R_{2}^{(P)}(\mathbf{s})
$$

Proof: The probability of success of the second relay in the setting with no information, $p_{2}^{(N)}(\mathbf{s})$, given in (18), can be rewritten as

$$
p_{2}^{(N)}(\mathbf{s})=e^{-\lambda s_{2}} q+\left(1-e^{-\lambda s_{2}}\right) p_{2}^{(P)}(\mathbf{s}),
$$

where $p_{2}^{(P)}(\mathbf{s})$, given in (14) is the probability of success of the second relay when it has partial information, and $q$, given in (10), is the probability of success of the first relay. The intuitive explanation of this relation is that the second relay does not know whether it is first or second. So, it will condition on when the other relay meets the source to determine the order. With probability $\left(1-e^{-\lambda s_{2}}\right)$, the other relay will have met the source before $s_{2}$. So, the second relay will correctly assume that it is second and use the probability of success with partial information because it does not know $s_{1}$. With probability $e^{-\lambda s_{2}}$, the other relay will meet the source after $s_{2}$. So, the second relay will wrongly assume that it is first and use the corresponding probability of success.

Also, $q \geq p_{2}^{(P)}(\mathbf{s})$, since the probability of success of the first relay is always greater than that of the second relay. Hence,

$$
p_{2}^{(N)}(\mathbf{s}) \geq p_{2}^{(P)}(\mathbf{s})
$$

Since the expected reward proposed to the second relay depends inversely on the probability of success, the claimed result follows.

Proposition 5 says that between the choice of informing a relay that is the second one and not giving this information, it is better for the source not to give this information.

Finally, let us compare the settings of full information with that of no information to determine which one is better for the source. For this comparison, we shall again compare the success probabilities in the two settings. Let us define the difference of the success probabilities in the two settings as

$$
g\left(v, s_{1}\right)=p_{2}^{(N)}\left(s_{1}, v+s_{1}\right)-p_{2}^{(F)}\left(s_{1}, v+s_{1}\right),
$$

where $v=s_{2}-s_{1}$. So, at $s_{2}=s_{1}+v$, if $g\left(v, s_{1}\right)<0$, then it is better for the source to give information. Otherwise, it is better for the source not to give information. We can rewrite $g\left(v, s_{1}\right)$ as $g\left(v, s_{1}\right)=a\left(s_{1}\right) e^{-\mu v}-b\left(s_{1}\right) e^{-\lambda v}$, where

$$
\begin{aligned}
& a\left(s_{1}\right)=\frac{1}{2}\left(\frac{\lambda}{\lambda-\mu} e^{-\mu s_{1}}-1\right), \text { and } \\
& b\left(s_{1}\right)=\frac{\mu^{2}}{\lambda^{2}-\mu^{2}} e^{-\lambda s_{1}},
\end{aligned}
$$

First let us consider the case $\lambda>\mu$.

Theorem 1: For $\lambda>\mu$, there exist $0 \leq \theta_{1} \leq \theta_{2}<\infty$ such that

1) if $0 \leq s_{1} \leq \theta_{1}$, then $g\left(v, s_{1}\right) \geq 0, \forall v \geq 0$;

2) if $s_{1} \geq \theta_{2}$, then $g\left(v, s_{1}\right)<0, \forall v \geq 0$;

3) if $\theta_{1}<s_{1}<\theta_{2}$, then

a) $g\left(v, s_{1}\right)<0, \forall s_{2} \in\left[s_{1}, s_{1}+\omega\left(s_{1}\right)\right)$

b) $g\left(v, s_{1}\right)>0, \forall s_{2} \in\left(s_{1}+\omega\left(s_{1}\right), \infty\right)$;

where

$$
\begin{aligned}
\theta_{2} & =-\frac{1}{\mu} \log \left(1-\frac{\mu}{\lambda}\right), \\
\omega\left(s_{1}\right) & =\frac{1}{\lambda-\mu} \log \left(\frac{b\left(s_{1}\right)}{a\left(s_{1}\right)}\right),
\end{aligned}
$$

and $\theta_{1}$ is the solution of $a\left(\theta_{1}\right)=b\left(\theta_{1}\right)$. Moreover, $\omega$ is an increasing and convex function.

Before going into the proof of the above result, some explanation of its consequences is in order. If the source met the first relay at $s_{1} \leq \theta_{1}$, then irrespective of the time instant at which it meets the second relay, it should not give any information to the second relay. On the other hand, if $s_{1} \geq \theta_{2}$, then it should give full information to the second relay irrespective of $s_{2}$. For $\theta_{1} \leq s_{1} \leq \theta_{2}$, then the strategy of the source should be threshold type : if it meets the second relay before $s_{1}+\omega\left(s_{1}\right)$, then it should give full information, otherwise it should not give any information. This is illustrated in Figure 1.

Proof of Theorem 1: First, note that for $s_{1} \geq \theta_{2}, a\left(s_{1}\right) \leq$ 0 which immediately shows that $g\left(v, s_{1}\right)<0, \forall v \geq 0$ thereby proving statement 2$)$.

For $s_{1}<\theta_{2}, a\left(s_{1}\right)>0$, and there is exactly one real solution of $g\left(v, s_{1}\right)=0$ seen as an equation in $v$, and this is given by $\omega\left(s_{1}\right)$. Moreover, at $v=\omega\left(s_{1}\right)$,

$$
\left.\frac{d}{d v} g\left(v, s_{1}\right)\right|_{v=\omega\left(s_{1}\right)}=(\lambda-\mu) b\left(s_{1}\right) e^{-\lambda \omega\left(s_{1}\right)}>0 .
$$




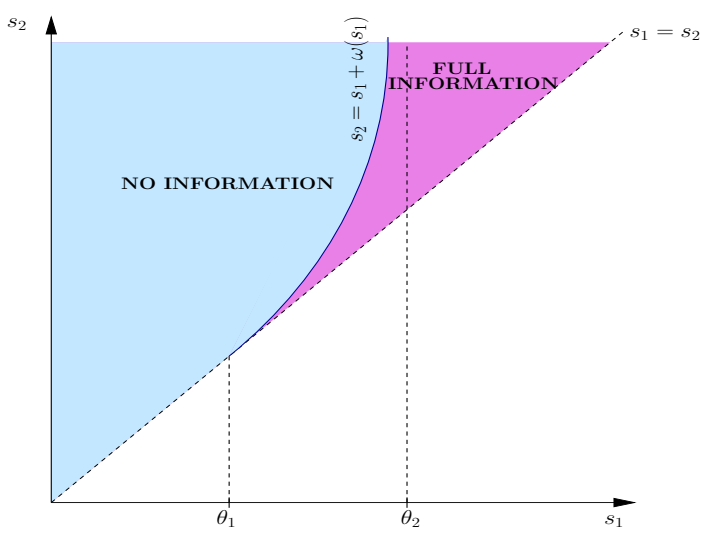

Fig. 1. Optimal strategy for the source for $\lambda>\mu$.

Hence, $g\left(v, s_{1}\right)<0$ for $v<\omega\left(s_{1}\right)$, and $g\left(v, s_{1}\right)>0$ otherwise.

To complete the proof, we need to show that $\omega\left(s_{1}\right)>0$ if and only if $s_{1}>\theta_{1}$. For this, note that

$$
\frac{d}{d s_{1}} \omega\left(s_{1}\right)=\frac{\lambda}{\lambda-\mu} \frac{1-e^{-\mu s_{1}}}{2 a\left(s_{1}\right)}>0,
$$

that is, $\omega\left(s_{1}\right)$ is a strictly increasing function of $s_{1}$. From $a\left(\theta_{1}\right)=b\left(\theta_{1}\right)$, we have $\omega\left(\theta_{1}\right)=0$. As a consequence $\omega\left(s_{1}\right)>$ 0 if and only if $s_{1}>\theta_{1}$.

The convexity of $\omega\left(s_{1}\right)$ follows from the fact that

$$
\frac{d^{2}}{d s_{1}^{2}} \omega\left(s_{1}\right)=\frac{\lambda \mu^{2}}{4(\lambda-\mu)^{2}} \frac{e^{-\mu s_{1}}}{\left(a\left(s_{1}\right)\right)^{2}}>0 .
$$

The other case $\lambda \leq \mu$ is similar with the difference that $\theta_{2}=\infty$. For any $s_{1}$ there will always be some values of $s_{2}$ when the source will not give information to the second relay. We state the result for this case without proof as it follows a similar reasoning to that of Theorem 1 .

Theorem 2: For $\lambda \leq \mu$, there exist $0 \leq \theta_{1}<\infty$ such that

1) if $0 \leq s_{1} \leq \theta_{1}$, then $g\left(v, s_{1}\right) \geq 0, \forall v \geq 0$;

2) if $\theta_{1}<s_{1}<\infty$, then

a) $g\left(v, s_{1}\right)<0, \forall s_{2} \in\left[s_{1}, s_{1}+\omega\left(s_{1}\right)\right)$;

b) $g\left(v, s_{1}\right)>0, \forall s_{2} \in\left(s_{1}+\omega\left(s_{1}\right), \infty\right)$;

where $\theta_{1}$ and $\omega\left(s_{1}\right)$ are as defined in Theorem 1.

This strategy for the source is illustrated in Figure 2. As a special case, for $\lambda=\mu$,

$$
\begin{aligned}
\theta_{1} & =\frac{-L W\left(-e^{-1.5}\right)-1.5}{\lambda}, \\
\omega\left(s_{1}\right) & =\frac{2 e^{\lambda s_{1}}-\left(3+2 \lambda s_{1}\right)}{2 \lambda},
\end{aligned}
$$

where $L W$ is the LambertW function.

\section{A. Adaptive versus Static strategies}

It was observed that the source can reduce the reward it has to pay by adapting its strategy depending on $s_{1}$ and $s_{2}$. Let

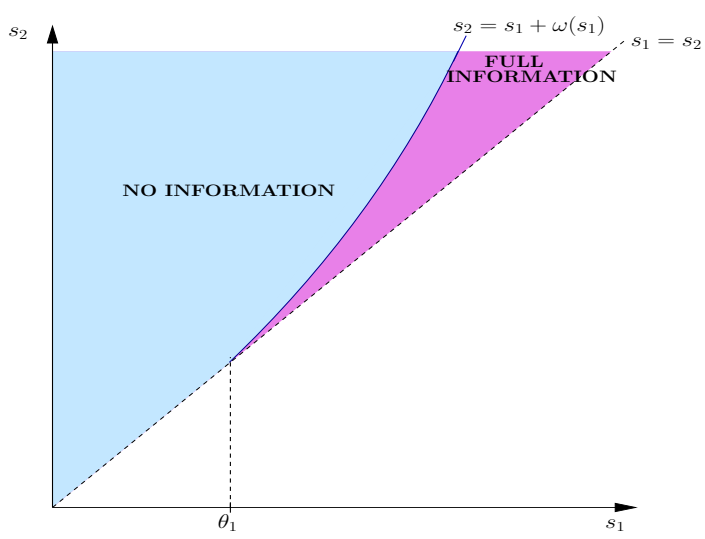

Fig. 2. Optimal strategy for the source for $\lambda<\mu$.

$\bar{R}^{(A)}$ denote the expected reward paid by the source when it uses the adaptive strategy. Then,

$$
\begin{aligned}
\bar{R}^{(A)} & =\int_{0}^{\infty} \int_{s_{2}=s_{1}}^{\infty}\left(R_{1}^{(F)}\left(s_{1}\right)(1-p(\mathbf{s}))+\right. \\
& \left.\min \left(R_{2}^{(F)}(\mathbf{s}), R_{2}^{(N)}\left(s_{2}\right)\right) p(\mathbf{s})\right) f_{S_{1}, S_{2}}(\mathbf{s}) d s_{2} d s_{1}
\end{aligned}
$$

Since the source has to pay at least $C_{1}+C_{2}$, it follows that Proposition 6:

$$
C_{1}+C_{2} \leq \bar{R}^{(A)} \leq \bar{R}^{(F)}=C_{1}+2 C_{2}
$$

Corollary 1:

$$
\frac{\bar{R}^{(A)}}{\bar{R}^{(F)}} \geq \frac{C_{1}+C_{2}}{C_{1}+2 C_{2}} \geq \frac{1}{2} .
$$

By using an adaptive strategy, the source can reduce its expenses at most by a factor of 0.5. Though the source can reduce the expected reward it pays to the relays, the difference $\bar{R}^{(F)}-\bar{R}^{(A)}$ is in fact borne by the relays. Thus, the relays do not have zero net expected cost when the source uses the adaptive strategy, and in fact incur a loss when the source adapts its strategy. In this case, the relays also have an incentive to adapt their strategy and ask for a higher reward. We do not model this strategic behaviour of the relays in the present work.

The exact analytical expression of $\bar{R}^{(A)}$ is difficult to compute unlike the expression for $\bar{R}^{(F)}$. In order to provide a quantitative comparison between the adaptive and the static settings, we resort to numerical experiments. In Figures 3 and $4, \bar{R}^{(A)}$ is plotted as a function of $\lambda$ for $\mu=1, C_{1}=1$, and $C_{2}=5\left(C_{2}=0.5\right.$ in Figure 4). It is observed that $\bar{R}^{(A)}$ increases with $\lambda$ and is gets close to $\bar{R}^{(F)}$ when $\lambda \rightarrow \infty$. On the other hand, for small values of $\lambda, \bar{R}^{(A)}$ is close to the minimal reward $C_{1}+C_{2}$. It appears that $\bar{R}^{(A)}$ has the form $\left(C_{1}+C_{2}\right)+C_{2}\left(1-e^{-\lambda \gamma}\right)$, for some constant $\gamma$, but we are unable to prove this result. 


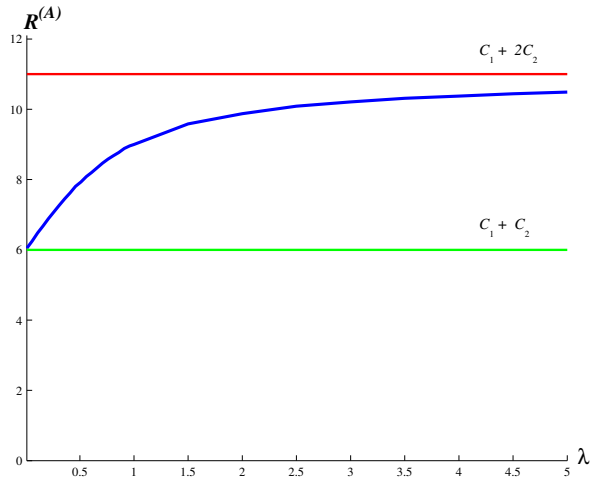

Fig. 3. Expected reward paid by the source for the adaptive statergy. $\mu=1$, $C_{1}=1, C_{2}=5$.

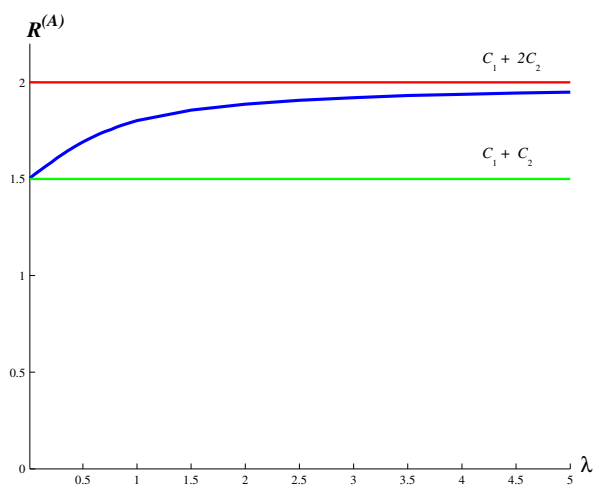

Fig. 4. Expected reward paid by the source for the adaptive statergy. $\mu=1$, $C_{1}=1, C_{2}=0.5$.

\section{CONCLUSIONS AND FUTURE WORK}

We investigated the impact of state information on the expected reward the source has to pay to relays in a twohop DTN. Three possible settings were considered: (i) full information; (ii) partial information; and (iii) no information. It was shown that on an average the source paid out the same expected reward in the three settings. The source can however reduce the expected reward by adapting the information as a function of the contact times with the relays. It was shown that it is optimal to always tell the first relay that it is the first one. For the second relay, a threshold type strategy is optimal, where the threshold depends on the contact time of the first relay. If the second relay arrives before this threshold, then it is optimal to give full information to it, otherwise it is optimal not to give any information.

The results obtained in this work are based on certain assumptions and simplifications that could be debated. The key assumption made in the definition of the adaptive strategy is that the second relay does not adapt the way it computes its success probability when it receive no information from the source. The fact that the source does not give information to the second relay could lead it to believe that the first relay met the source at a time further away in the past than what is suggested by the exponential distribution. The second relay could thus ask for a higher reward than in the no information setting, and offset any potential gains of the adaptive strategy. Another assumption made relates to the distribution of the contact process with the source and the destination. These were assumed to be exponentially distributed. While this assumption has been experimentally verified under certain circumstances and widely used as a model for the inter-contact times, other distributions such as heavy-tailed distributions have also been proposed as a model for the contact process. A final remark concerns the number of relays in the network. The current work assumes that there are two relays which leads to a simpler analysis.

Our current work focusses on relaxing these assumptions, with emphasis being placed on modelling the strategic response of the second relay to the adaptive strategy of the source. The objective would be to determine an equilibrium strategy from which neither the source nor the relays have an incentive to deviate.

\section{REFERENCES}

[1] X. Zhang, G. Neglia, J. Kurose, and D. Towsley, "Performance modeling of epidemic routing," Elsevier Computer Networks, vol. 51, no. 10, pp.2867-2891, 2007.

[2] E. Altman, F. De Pellegrini, and L. Sassatelli, "Dynamic control of coding in delay tolerant networks," in Proc. of IEEE INFOCOM, San Deigo, USA, March 15-17, 2010.

[3] R. El-Azouzi, F. De Pellegrini, H. B. Sidi, and V. Kamble, "Evolutionary forwarding games in delay tolerant networks: Equilibria, mechanism design and stochastic approximation," Computer Networks, 2012. [Online]. Available: http://www.sciencedirect.com/science/article/pii/S1389128612003982

[4] R. El-Azouzi, H. B. Sidi, F. De Pellegrini, and Y. Hayel, "Markov decision evolutionary game for energy management in delay tolerant networks," in NETGCOOP 2011, October, 2011.

[5] E. Altman, A. P. Azad, T. Basar, and F. De Pellegrini, "Optimal activation and transmission control in delay tolerant networks," in Proc. of IEEE INFOCOM, San Deigo, USA, March 15-17, 2010, extended version available on ArXiv.

[6] T. Karagiannis, J.-Y. L. Boudec, and M. Vojnovic, "Power law and exponential decay of intercontact times between mobile devices," IEEE Trans. Mob. Comput., vol. 9, no. 10, pp. 1377-1390, 2010.

[7] U. Shevade, H. Song, L. Qiu, and Y. Zhang, "Incentive-aware routing in dtns," in Network Protocols, 2008. ICNP 2008. IEEE International Conference on. IEEE, 2008, pp. 238-247.

[8] B. Chen and M. Chan, "Mobicent: a credit-based incentive system for disruption tolerant network," in INFOCOM, 2010 Proceedings IEEE. IEEE, 2010, pp. 1-9.

[9] L. Wei, Z. Cao, and H. Zhu, "Mobigame: A user-centric reputation based incentive protocol for delay/disruption tolerant networks," in Global Telecommunications Conference (GLOBECOM 2011), 2011 IEEE. IEEE, 2011, pp. 1-5.

[10] Q. Li, S. Zhu, and G. Cao, "Routing in socially selfish delay tolerant networks," in INFOCOM, 2010 Proceedings IEEE. IEEE, 2010, pp. $1-9$.

[11] M. Y. S. Uddin, B. Godfrey, and T. Abdelzaher, "Relics: In-network realization of incentives to combat selfishness in dtns," in Network Protocols (ICNP), 2010 18th IEEE International Conference on. IEEE, 2010, pp. 203-212.

[12] Y. Wang, M.-C. Chuah, and Y. Chen, "Incentive driven information sharing in delay tolerant mobile networks," in Global Communications Conference (GLOBECOM), 2012 IEEE. IEEE, 2012, pp. 5279-5284.

[13] W. Chahin, H. B. Sidi, R. El-Azouzi, F. De Pellegrini, and J. Walrand, "Incentive mechanisms based on minority games in heterogeneous dtns," 25th ITC conference, Sanghai, China, Sept. 10-12, 2013.

[14] R. W. Wolff, Stochastic modeling and the theory of queues, ser Prentice-Hall international series in industrial and systems engineering. Englewood Cliffs, N.J. Prentice Hall, 1989. 